\title{
$\mathrm{HEVC}$ 에서 시공간적 상관관계를 이용한 엔트로피 부호화 방법
}

\author{
김 태 룡 ${ }^{a}$, 김 경 용 ${ }^{a)}$, 이 한 수 ${ }^{a)}$, 박 광 훈 ${ }^{a}$
}

\section{A Entropy Coding Method using Temporal and Spatial Correlation on HEVC}

\author{
Tae Ryong Kim ${ }^{\text {a)}}$, Kyung Yong Kim ${ }^{\text {a) }}$, Han Soo Lee ${ }^{\text {a) }}$, and Gwang Hoon Park ${ }^{\text {a) }}$ \\ 요 약
}

$\mathrm{CU}$ 정보 중에서 분할 정보 및 스킵 정보는 공간적으로 인접한 $\mathrm{CU}$ 뿐만 아니라 시간적으로 대응되는 $\mathrm{CU}$ 와 매우 유사한 특성을 지 닌다. 본 논문은 $\mathrm{CU}$ 정보를 부호화할 때, 공간적 상관도뿐만 아니라 시간적 상관도를 이용함으로써 부호화 효율을 향상시키는 방법을 제안한다. $\mathrm{CABAC}$ 의 경우에는 $\mathrm{CU}$ 분할정보 및 스킵 정보에 대한 문맥모델을 생성할 때 시간적으로 대응되는 $\mathrm{CU}$ 의 정보를 활용하 고, CAVLC의 경우에는 시간적으로 대응되는 CU정보를 이용하여 현재 $\mathrm{CU}$ 정보들을 유추하는 방법을 이용하여 부호화하였다. 그 결 과 현재까지 나온 $\mathrm{HM} 3.0$ 와 비교하여 $\mathrm{CABAC}$ 에서 $0.1 \% \sim 0.6 \%$ 의 성능 향상을 보였고 CAVLC에서는 $0.1 \% \sim 0.4 \%$ 의 성능 향상이 있었 고, 특히 시간적으로 가까운 참조프레임을 사용하는 저지연 환경에서 더 좋은 성능을 보였다.

\begin{abstract}
The split flag and the skip flag in CU syntax have high correlation on spatial domain as well as temporal domain. This paper suggests a method for enhancing coding efficiency by using not only spatial correlation but also temporal correlation when coding $\mathrm{CU}$ information. In the CABAC case, temporal collocated $\mathrm{CU}$ information is used for selecting context model of the split flag and the skip flag. In the CAVLC case, current $\mathrm{CU}$ information is estimated from temporal collocated $\mathrm{CU}$ information then encoded. As a result, a coding efficiency was increased by $0.1 \% \sim 0.6 \%$ in CABAC, $0.1 \% \sim 0.4 \%$ in CAVLC compared with HM 3.0. This method shows better performance on lowdelay condition which uses reference frame close to current frame.
\end{abstract}

Keyword : HEVC, Entropy Coding, CABAC, CAVLC

\section{I. 서 론}

현재 사용되는 동영상 압축 표준인 H.264/AVC의 기술 이 앞으로 사용자들에게 제공할 $\mathrm{UHD}$ 급의 영상을 다루기

a) 경희대학교 전자정보대학 컴퓨터공학과

Dept. of Computer Engineering, College of Electronics and Information, Kyung Hee University

\# 교신저자 : 박광훈 (ghpark@khu.ac.kr)

※ 본 연구는 지식경제부 및 정보통신산업진흥원의 대학 IT연구센터 지원 사업의 연구결과로 수행되었음 (NIPA-2011-(C1090-1111-0001))

· 접수일(2011년10월10일), 수정일(2011년12월13일),게재확정일(2011년12월13일)
에는 압축률이나 처리 속도 측면에 있어서 부족한 부분이 많다고 판단하여 차세대 동영상 압축 표준인 $\mathrm{HEVC}(\mathrm{High}$ Efficiency Video Coding $)^{[2]}$ 개발에 착수 하였다. HEVC에 서는 영상의 특성에 따라 적응적으로 변화가 가능한 다양 한 크기의 정사각형 $\mathrm{CU}($ Coding Unit) 단위를 사용하고 있 다 ${ }^{[2]}$. CU는 가장 큰 단위인 LCU(Largest Coding Unit)에서 가장 작은 단위인 $\mathrm{SCU}(\mathrm{Smallest}$ Coding Unit)까지 분할 될 수 있으며, $\mathrm{CU}$ 의 분할 여부는 각 $\mathrm{CU}$ 마다 저장된 분할 정보 (split information)를 통해 알 수 있다. 이러한 CU의 분할정 보는 공간적 뿐만 아니라 시간적으로도 매우 유사한 특성 
을 지닌다. 따라서 본 논문에서는 시공간적인 상관도를 이 용하여 효율적으로 부호화하는 방법을 제안한다.

본 논문의 구성은 다음과 같다. ㅍㅈ장에서는 $\mathrm{HEVC}$ 의 엔 트로피 부호화 기술과 효율 개선을 제안하는 배경을 설명 하고 피장에서는 효율적인 엔트로피 부호화 방법을 제안한 다. IV장에서는 제안한 방법에 대한 성능을 보이고 마지막 $\mathrm{V}$ 장에서는 결론을 도출하고 향후 발전해 나갈 수 있는 방 향에 대해서 모색한다.

\section{HEVC에서의 엔트로피 부호화 기술 방법과 제안 배경}

$\mathrm{HEVC}$ 에서 사용하는 엔트로피 부호화 방법은 문맥 기반 적응적 산술부호화 $\left(\mathrm{CABAC}^{[2]}\right)$ 와 문맥 기반 적응적 가변길 이 부호화 $\left(\mathrm{CAVLC}^{[2]}\right)$ 로 나눌 수 있다. 두 방법은 주로 공간 적인 상관도만을 이용하여 엔트로피 부호화를 수행한다. 하지만 $\mathrm{CU}$ 의 분할 정보와 스킵 정보의 경우에는 시간적으 로도 밀접한 상관관계가 존재한다. <그림 $1>$ 은 $\mathrm{HEVC}$ 실 험 영상으로 분할 정보와 스킵 모드정보(skip information) 의 시공간적인 상관도를 보여주는 그림이다.

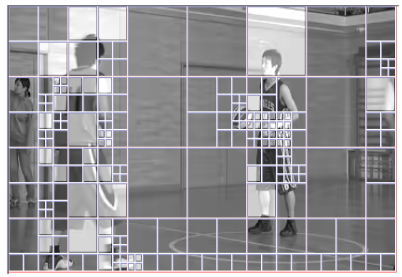

(a) BasketballPass, Frame: 1

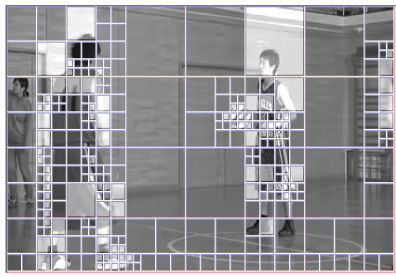

(b) BasketballPass, Frame: 2
그림 1. 분할 정보와 스킵 정보의 시공간적인 상관도

Fig. 1. spatio-temporal correlation of split flag and skip flag

<그림 1>에서 사각형 블록은 $\mathrm{CU}$ 를 나타내며 영상의 특 성에 따라 큰 크기의 $\mathrm{CU}$ 혹은 작은 $\mathrm{CU}$ 의 크기로 분할된 것을 확인할 수 있다. 여기서 <그림 $1(\mathrm{a})>$ 의 과거 영상과 <그림 1(b)>에서 시간적으로 인접한 두 개의 영상에서 서 로 대응하는 $\mathrm{CU}$ 의 분할 정도가 유사한 것은 분할 정보의 시간적인 상관도가 높다는 것을 보여준다. 또한 <그림 1>
의 어두운 사각형 블록은 스킵 모드(skip mode)로 부호화된 $\mathrm{CU}$ 를 나타내는데, 시간적으로 인접한 두 개의 영상에서 서 로 대응하는 어두운 블록들이 유사한 것을 확인할 수 있다. 이는 스킵 모드의 부호화 정보 역시 시간적인 상관도가 있 음을 보여준다.

\section{III. 제안하는 HEVC 엔트로피 부호화 기술}

$\mathrm{CU}$ 분할 정보와 스킵 모드정보의 경우에 시간적 상관도 가 존재하므로 이러한 특성을 이용하여 엔트로피 부호화를 수행할 수 있다. CU 분할 정보와 스킵 모드정보가 아닌 다 른 부호화 정보들은 공간적 상관관계에 영향을 많이 받지 않는다. ${ }^{[6]}$ 따라서 본 논문에서는 분할 정보와 스킵 모드정 보에 한하여 시공간적인 상관도를 고려한 엔트로피 부호화 방법을 제안한다.

1. CABAC에서의 제안하는 부호화 방법

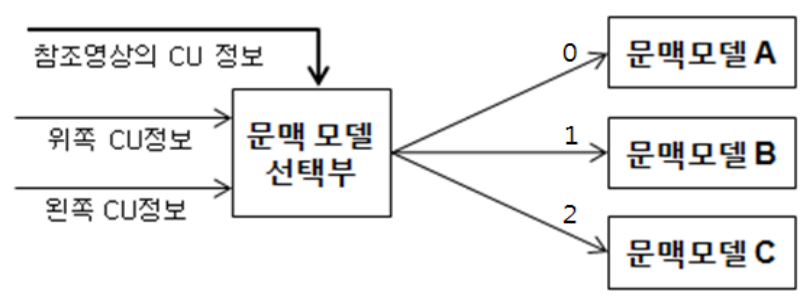

그림 2. 제안하는 문맥 모델 선택 방법

Fig. 2. Proposed method for selecting context model

<그림 2>는 $\mathrm{CABAC}$ 에서 참조영상에서 현재 $\mathrm{CU}$ 에 대응 되는 $\mathrm{CU}$ 정보를 추가로 고려하여 문맥 모델을 선택하는 방 법을 나타낸다. 분할 정보를 부호화하기 위해 문맥 모델을 선택할 때 시간적 상관도의 크기가 공간적 상관도보다 크 기 때문에 문맥 모델을 선택할 때 우선순위를 시간적 상관 도에 두며 그 방법은 아래와 같다.

1) $\mathrm{Ctx}$ 는 문맥 모델을 선택하는 인덱스를 의미하며 0 으로 초기화 한다.

2) 참조영상에서 대응하는 $\mathrm{CU}$ 의 분할 정보를 보고, 분할된 경우 
문맥 모델을 선택하는 인덱스를 +1 시킨다.

3) 현재 부호화 하려는 $\mathrm{CU}$ 와 인접한 위쪽 $\mathrm{CU}$ 의 분할정보와 왼쪽 $\mathrm{CU}$ 의 분할정보를 살펴보고 분할이 되었을 경우마다 문맥 모델 을 선택하는 인덱스를 +1 을 시킨다. 단, 문맥 모델을 선택하는 인덱스가 2 보다 큰 경우에는 2 로 고정시킨다.

4) 모델을 선택하는 인덱스에 따라 문맥모델이 선택된다.

스킵 모드정보를 부호화하기 위해 문맥 모델을 선택하는 방법은 분할 정보와 동일한 방법으로 수행된다.

\section{CAVLC에서의 제안하는 부호화 방법}

CAVLC에서 제안하는 방법은 CABAC과 동일하게 인터 예측 $\mathrm{CAVLC}^{[2]}$ 테이블에 포함된 분할 정보와 스킵 모드정 보에 한해서만 제안되는 방법으로 인트라 예측에서 사용되 는 MPM(Most Probable Mode) ${ }^{[1]}$ 과 유사한 방법을 이용하 여 시간적 및 공간적 상관도를 적응적으로 적용시켰다. <그 림 3>의 제안하는 방법을 살펴보면 기존의 CAVLC 테이블 을 갱신하는 방법을 유지하며 특정 조건에서만 플래그(flag) 정보를 추가하여 보냄으로써 효율적인 부호화를 수행한다.

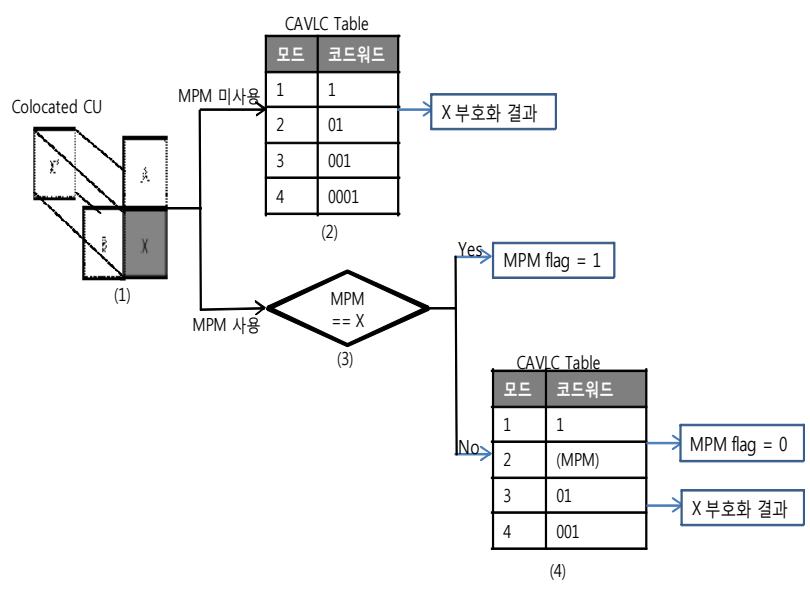

그림 3. 제안하는 CAVLC 부호화 방법

Fig. Proposed method for CAVLC

<그림 3(1)>은 $\mathrm{CU}$ 들을 표현한 것이며 각 $\mathrm{CU}$ 내에 $\mathrm{A}$, $\mathrm{B}, \mathrm{X}^{\prime \prime}, \mathrm{X}$ 는 특정 모드를 임의로 표현한 것이다. 제안하는 방법은 현재 $\mathrm{CU}$ 에서 $\mathrm{X}$ 모드를 부호화하기 전에 시공간적 으로 인접한 $\mathrm{CU}$ 정보를 보고 현재 $\mathrm{CU}$ 가 부호화 될 가장
높은 확률을 예측한 모드인 MPM의 사용여부를 결정하는 데 그 방법은 아래와 같다.

1) 분할 정보의 문맥 모델을 선택하는 인덱스와 스킵 모드정보의 문맥 모델을 선택하는 인덱스를 구한다. 이때 인덱스를 구하는 방법은 $\mathrm{CABAC}$ 에서 제안한 문맥 모델을 선택하는 방법을 이 용한다.

2) 두 개의 문맥 모델 인덱스 중 2 의 값이 존재하면 MPM을 사용 하며 그렇지 않으면 사용하지 않는다.

<그림 3(2)>는 MPM이 사용되지 않을 경우 부호화 되는 과정을 표현한 것으로 부호화 방법은 HM $3.0^{[3]}$ 의 CAVLC 와 동일한 방법으로 수행된다. <그림 3(3)>은 MPM을 사 용하여 부호화하는 과정이며, 여기서는 예측되어진 MPM 이 실제로 부호화 되는 X모드와 동일한 모드인지를 판단 하는 과정이다. 이 과정에서 MPM과 X 모드가 동일한 모드이면 1 비트의 MPM 플래그만을 부호화 한다. <그림 3(4)>는 MPM과 X모드가 동일하지 않았을 경우 부호화되 는 과정을 표현한 것으로, 기존의 CAVLC 테이블에서 MPM 모드를 생략한 테이블을 이용하여 부호화를 수행하 며 1 비트의 MPM 플래그와 X모드를 테이블을 이용하여 부호화한다.

\section{V. 제안하는 방법의 성능 평가}

본 논문에서 제안하는 방법의 성능을 평가하기 위해 $\mathrm{HEVC}$ 의 참조 소프트웨어(reference software)인 $\mathrm{HM} 3.0^{[3]}$ 에 실제 구현을 하여 기존의 $\mathrm{CABAC}$ 와 $\mathrm{CAVLC}$ 의 부호화 성능을 비교하였다. 실험에 사용된 영상은 현재 $\mathrm{HEVC}$ 의 표준화 작업에 사용되는 테스트 영상ㅍㅇ이며, 실험은 인코딩 시간과 디코딩 시간을 측정하기 위해 동일한 환경의 운영 체제와 성능으로 수행하였다. 기본적인 실험 조건은 $\mathrm{HEVC}$ 공통실험조건 ${ }^{[4]}$ 을 이용하였다. 실험결과는 부호화 효율을 측정하기 위해 평균적인 bit-rate 감소량을 나타내는 BD-Bitrate ${ }^{[5]}$ 방법을 사용하였다. 아래의 <표 1>는 제안하 는 엔트로피 부호화 방법에 대한 결과이다. 
표 1. 제안방법의 실험 결과

Table 1. Experimental result of the proposed method

\begin{tabular}{|c|c|c|c|c|c|c|}
\hline & \multicolumn{3}{|c|}{ Random access } & \multicolumn{3}{|c|}{ Random access LoCo } \\
\hline & Y BD-rate & UBD-rate & VBD-rate & Y BD-rate & U BD-rate & VBD-rate \\
\hline Class A & -0.1 & -0.2 & -0.4 & -0.1 & -0.1 & -0.1 \\
\hline Class B & -0.1 & -0.3 & -0.1 & -0.1 & -0.1 & -0.1 \\
\hline Class C & -0.1 & -0.1 & 0.0 & -0.1 & -0.1 & -0.1 \\
\hline Class D & -0.1 & -0.1 & -0.1 & -0.1 & -0.1 & -0.1 \\
\hline \multicolumn{7}{|l|}{ Class $\mathrm{E}$} \\
\hline All & -0.1 & -0.2 & -0.1 & -0.1 & -0.1 & -0.1 \\
\hline Enc Time[\%] & \multicolumn{3}{|c|}{$101 \%$} & \multicolumn{3}{|c|}{$101 \%$} \\
\hline Dec Time[\%] & \multicolumn{3}{|c|}{$102 \%$} & \multicolumn{3}{|c|}{$102 \%$} \\
\hline & \multicolumn{3}{|c|}{ Low delay } & \multicolumn{3}{|c|}{ Low delay LoCo } \\
\hline & Y BD-rate & U BD-rate & VBD-rate & Y BD-rate & UBD-rate & V BD-rate \\
\hline \multicolumn{7}{|c|}{ 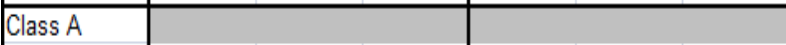 } \\
\hline Class B & -0.1 & 0.0 & 0.1 & -0.2 & -0.2 & -0.2 \\
\hline Class C & -0.2 & -0.4 & 0.1 & -0.2 & -0.2 & -0.2 \\
\hline Class D & -0.2 & -0.2 & 0.0 & -0.2 & -0.2 & -0.2 \\
\hline Class E & -0.6 & -0.6 & -0.5 & -0.4 & -0.4 & -0.4 \\
\hline All & -0.3 & -0.2 & 0.0 & -0.2 & -0.2 & -0.2 \\
\hline Enc Time[\%] & \multicolumn{3}{|c|}{$102 \%$} & \multicolumn{3}{|c|}{$102 \%$} \\
\hline Dec Time[\%] & \multicolumn{3}{|c|}{$102 \%$} & \multicolumn{3}{|c|}{$102 \%$} \\
\hline
\end{tabular}

제안한 방법을 이용하여 엔트로피 부호화를 수행한 결과 $<$ 표 1>과 같이 모든 클래스에서 전체적인 성능 향상을 확 인 할 수 있다. 'Low delay' 조건에서 영상별 부호화 효율을 보았을 때 모든 영상에서 $0.1 \% \sim 0.6 \%$ 의 성능 향상이 있었 고, 'Random access'조건에서 역시 모든 영상에서 $0.1 \%$ 정 도의 성능 향상을 볼 수 있었다. 여기서 'Low delay'의 부호 화 성능이 'Random access'와 비교하여 부호화 효율이 더 좋은 것을 확인할 수 있다. 이는 'Low delay'조건의 경우 참조영상이 시간 축으로 바로 이전의 영상을 사용함으로써 시간적인 상관도가 높은 반면 'Random access'조건의 경우 'Low delay'조건과 비교하여 참조영상이 시간적으로 멀리 떨어진 경우가 존재하여 시간적 상관도가 비교적 낮았기 때문이다. 또한 'Low delay'조건에서 $\mathrm{E}$ 클래스의 결과가 다 른 클래스에 비해 높은 효율을 얻은 것을 볼 수 있다. 이러 한 결과는 $\mathrm{E}$ 클래스의 영상 특성상 배경의 변화가 적고 움 직임이 복잡하지 않아서 시간적인 유사도가 높아 제안한 방법이 효율적으로 적용된 결과이다. 복잡도 측면에서 보 았을 때는 기존 $\mathrm{HM} 3.0$ 보다 CABAC과 CAVLC 모두 참조 하지 않았던 $\mathrm{CU}$ 블록을 참조하기 때문에 복잡도가 증가한 다. 그러나 제안한 방법이 차지하는 복잡도는 전체적인 부
호화와 복호화 시간에서 차지하는 부분에 있어 작은 부분 이기 때문에 부호화와 복호화 시간이 미미하게 증가하였다.

\section{VI. 결 론}

본 논문에서는 $\mathrm{HEVC}$ 에서 $\mathrm{CU}$ 의 정보들 중 분할정보와 스킵모드 정보에 한하여 엔트로피 부호화를 수행할 때 시 공간적인 상관도를 적응적으로 이용하여 효율적으로 부호 화하는 방법에 대해 제안하였다. 그 결과 동일한 수준의 복 잡도를 유지하면서 'Lowdelay'조건에서는 $0.1 \% \sim 0.6 \%$ 의 성능 향상을 볼 수 있고, 'Random access'조건에서는 $0.1 \%$ 의 성능향상을 확인 할 수 있었다. 그렇지만, 제안하는 방법 은 에러가 존재하는 환경에서 참조영상에 문제가 생기면 인코더와 디코더의 동기화 문제로 확산 될 수 있다. 따라서 주기적으로 공간적인 상관도만을 이용한 부호화를 하는 방 법과 같은 에러 확산을 방지하는 방법들이 필요하다. 또한 CAVLC에서는 필요하지 않았던 CU들을 참조하면서 의존 적인 파싱을 요구하며 추가적인 $\mathrm{CU}$ 정보들의 저장공간을 필요로 한다.

\section{참 고 문 헌}

[1] ITU-T Recommendation H.264 and ISO/IEC 14496-10 (MPEG-4 Part 10 AVC), "Advanced Video Coding for Generic Audiovisual Services," Version 1: March 2003, Version 2: May 2004, Version 3: March 2005, Version 4: September 2005, Version 5 and Version 6: June 2006, Version 7: April 2007, Version 8: July 2007.

[2] Joint Collaborative Team on Video Coding (JCT-VC) of ITU-T SG16 WP3 and ISO/IEC JTC1/SC29/WG11, "WD3: Working Draft 3 of High-Efficiency Video Coding", JCTVC-E603, Geneva, Switzerland, March 2011.

[3] https://hevc.hhi.fraunhofer.de/svn/svn_HEVCSoftware/

[4] Joint Collaborative Team on Video Coding (JCT-VC) of ITU-T SG16 WP3 and ISO/IEC JTC1/SC29/WG11, "Common test conditions and software reference configurations", JCTVC-E700, Geneva, Switzerland, March 2011.

[5] G. Bjøntegaard, "Calculation of average PSNR differences between RD-curves," ITU-T SG16 Q.6, VCEG-M33, Texas, USA, April 2001.

[6] Hisao Sasai, Takahiro Nishi, "Modified Context Derivation for Complexity reduction,” JCTVC-F429, Torino, IT, 14-22 July, 2011. 